\title{
A novel nonsense ATP2C1 mutation causes Hailey-Hailey disease in a Tunisian family
}

\section{Marwa Chourabi'1,2, Dorra H'mida-Ben Brahim², Carine Bonnard', Amina Aounallah', Alvin Yu Ng${ }^{4}$, Sumanty Tohari ${ }^{4}$, Byrappa Venkatesh ${ }^{4}$, Ali Saad ${ }^{2}$, Lobna Boussofara ${ }^{3}$, Bruno Reversade ${ }^{1}$, Mohamed Denguezli ${ }^{3}$}

\begin{abstract}
${ }^{1}$ Laboratory of Human Genetics and Embryology, Institute of Medical Biology, A*STAR, Singapore, ${ }^{2}$ Laboratory of Human Cytogenetic, Molecular Genetics and Reproductive Biology, Farhat Hached University Hospital, Sousse, Tunisia, ${ }^{3}$ Department of Dermatology and Venerology, Farhat Hached University Hospital, Sousse, Tunisia, ${ }^{4}$ Institute of Molecular and Cell Biology, $A^{*}$ STAR, Singapore
\end{abstract}

Corresponding author: Prof. Mohamed Denguezli, E-mail: denguezli@yahoo.fr

\begin{abstract}
Background: Hailey-Hailey disease (HHD) is an autosomal dominant blistering skin disorder that manifests in the third to fourth decade of life. The ATP2Cl has been identified as the pathogenic gene of this disease since 2000. Materials and Methods: We report here a three generations Tunisian pedigree, where almost all males are severely affected and present with complete penetrance of HHD, while only one female shows a mild disease's phenotype in her fourth decade. A molecular study using Whole exome sequencing and direct sequencing was performed to this family. Results: By whole exome sequencing and direct DNA sequencing, a novel nonsense mutation in ATP2Cl (c.2698A > T; p.Lys900Ter) was identified in all patients, supporting that alterations in ATP2Cl are causative of HHD. Unexpectedly, this mutation was found in one female who was initially not diagnosed for HHD. Our observations would be in line with incomplete penetrance and variable expressivity between male and female of this disease, or evidence for genetic modifiers. Conclusion: We report here a novel nonsense heterozygous mutation in ATP2Cl gene in 5 patients with HHD. Interestingly, one woman carries the nonsense ATP2Cl mutation but displays a mild phenotype of HHD. This could indicate a variation in pattern and expressivity between male and female developing HHD phenotype which should be considered when providing genetic counselling to family members carrying such mutations.
\end{abstract}

Keywords:ATP2C1; Hailey-Hailey disease; benign familial chronic pemphigus.

\section{INTRODUCTION}

Hailey-Hailey disease (HHD), or benign familial chronic pemphigus, which was described by the Hailey brothers in 1939, is characterized by recurrent vesicles and erosions, usually affecting the neck, axillae and groins [1]. HHD is an autosomal dominant blistering skin disorder that manifests in the $3^{\text {rd }}$ to $4^{\text {th }}$ decade of life with a prevalence of 1 in 50,000 [1,2]. Vesicular, itchy and painful lesions on an erythematous base characterize the rash and affect the flexural areas of the skin. Friction, sweating, heat, stress, UV exposure and cutaneous infections trigger the rash. The disease has a fluctuating course with remissions and exacerbations [3]. In rare instances, the rash may become generalized and the skin lesions may even develop into squamous cell carcinoma [4]. Penetrance in adults affected by HHD is complete but expressivity is variable [2]. Histopathological features show a widespread loss of cell-to-cell adhesion (acantholysis) in the suprabasal layer of the epidermis. Ultrastructural studies demonstrate perinuclear aggregation of keratin intermediate filaments, which have retracted from the desmosomal plaques in the acantholytic cells $[5,6]$.

Two different research groups have linked HHD to mutations in the gene ATP2C1, which encodes the human secretory pathway $\mathrm{Ca} 2+/ \mathrm{Mn} 2+$ ATPase

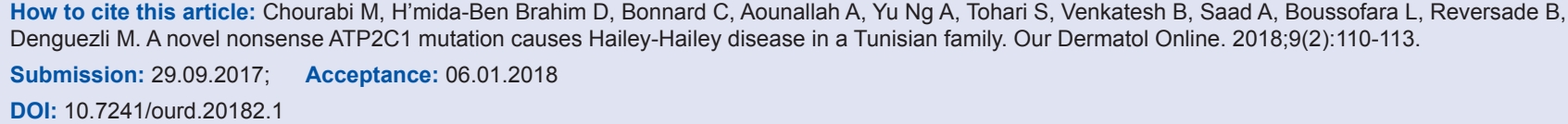


(hSPCAl) [7,8]. SPCAl is ubiquitously expressed in human tissues with the highest abundance in keratinocytes [9]. SPCAl localizes to the Golgiapparatus and controls its $\mathrm{Ca}^{2+}$ stores along with SERCA transporters. In primary keratinocytes, the role of SPCAl is more significant than in other cell types $[10,11]$, explaining why only the skin is affected in HHD patients. In this report we investigated a three generations Tunisian pedigree where males are severely affected with HHD. By whole exome sequencing followed by Sanger sequencing, we identified a novel nonsense mutation (c.2698A > T; p.Lys900Ter) in the ATP2Cl gene that causes Hailey-Hailey phenotype in this family

\section{MATERIALS AND METHODS}

\section{Subject and informed consent}

We performed molecular studies in a Tunisian family with HHD where males are severely affected in three generations (Fig. la). After obtaining the informed written consent and IRB approval (NUS IRB 10-051), blood samples were taken from four affected males and two unaffected females. Genomic DNA (gDNA) was extracted using Qiagen ${ }^{\circledR}$ kit.

\section{Whole exome sequencing}

For mutation analysis, lug of gDNA of proband III:8 was used for exome capture with Ion TargetSeqTM Exome and Custom Enrichment Kit. The exome library was prepared on an Ion OneTouch System and sequenced on an Ion Proton instrument (Life Technologies, Carlsbad, CA, USA) using one ION PI chip. Sequence reads were aligned to the human reference genome [Human GRCh37 (hg19) build] using Torrent Mapping Alignment Program (TMAP) from the Torrent Suite (v4.2.1). The variants were called using the Torrent Variant Caller (TVC) plugin (v4.2.1) and were annotated using the "annotate single sample variants" workflow, including the associated gene, variant location, quality-score, coverage, predicted functional consequences, protein position and amino acid changes, SIFT [12], PolyPhen2 [13], and Grantham [14] prediction scores, phyloP [15] conservation scores and 5000 genomes Minor Allele Frequencies. Annotated variants were filtered for common SNPs using the ClinVar "common and no known medical impacts" database (ftp://ftp.ncbi.nlm. nih.gov/pub/clinvar/vcf_GRCh37/) and the Exome
Aggregate Consortium (ftp://ftp.broadinstitute.org/ pub/ExAC_release/release0.2/). Variants were next compared to an in-house database of 485 previously sequenced samples, and those that were present in more than $1 \%$ of the previously sequenced samples were removed.

\section{Sanger sequencing}

Sanger sequencing was performed using 2 different sets of primers (Table 1) to verify the segregation of the identified ATPC2 nonsense mutation. After amplification, products were purified and sequenced on DNA sequencing system (model 3730XL; ABI).

\section{RESULTS AND DISCUSSION}

For all the affected members from this family (Fig. la) the disease began within the third decade of life. All affected individuals exhibited the typical clinical features of HHD with late onset of the rash characterized by itchy and painful lesions on an erythematous base and affect the flexural areas of the skin (Figs lb and c). All the patients mentioned that friction, heat or sweating exacerbated the disease and the symptoms were worse during summer or if they were under stress. It is noteworthy that HHD penetrance is complete in adults affected with a variable expressivity.

To look for the causative mutation, lug of gDNA of patient III:8 was used for exome capture with Ion TargetSeqTM Exome and Custom Enrichment Kit. Whole exome sequencing of this proband generated a total of $14.5 \mathrm{~Gb}$ with an average read length of $155 \mathrm{bp}$. An average coverage of $188 \mathrm{X}$ was achieved across the exome, with $96 \%$ of the targeted sequences covered at $\geq 20 X$. A total of 37,131 variants were identified across protein-coding exons, UTRs, splice sites and flanking introns. After applying a series of filters and following an autosomal dominant mode of inheritance, a final set of 28 heterozygous variants were selected (Fig. 2a), including a deleterious mutation in ATP2C1, known as the causative gene of HHD. By Sanger sequencing, we confirmed that the nonsense mutation c.2698A > T (p.Lys900Ter) was heterozygous in all affected male members and unexpectedly in the female III:10 who was initially not diagnosed for HHD (Fig. 2b). This result prompted us to re-examine this individual over time, and noted that she has started developing a milder HHD phenotype, less severe than her male siblings and father despite the fact that she is in 


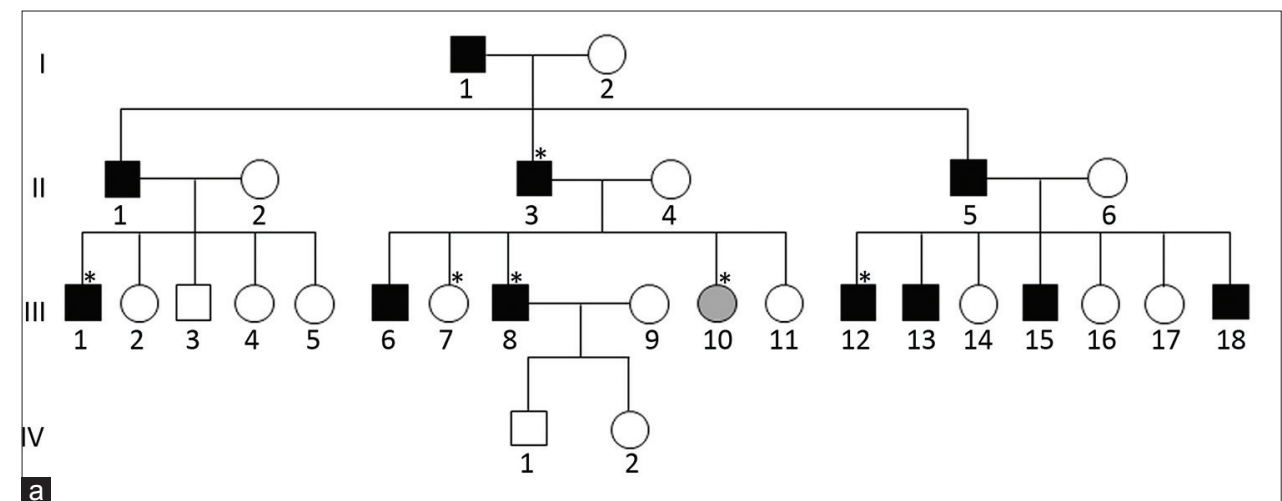

a

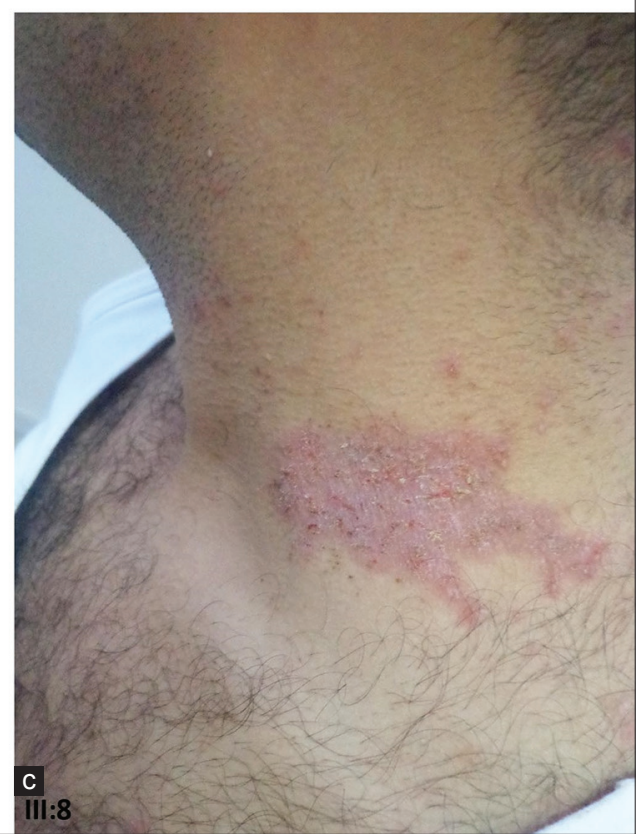

Figure 1: Clinical manifestations of autosomal dominant Hailey-Hailey disease. (a) Pedigree of a Tunisian family presents with an autosomal dominant HHD, where only males are severely affected on three generations (filled black symbols: affected individuals, square: male, circle: female, stars: studied individuals). (b) and (c) patients II:3 and III:8 with HHD features: rash that is characterized by painful lesions affecting the neck and the axillary.

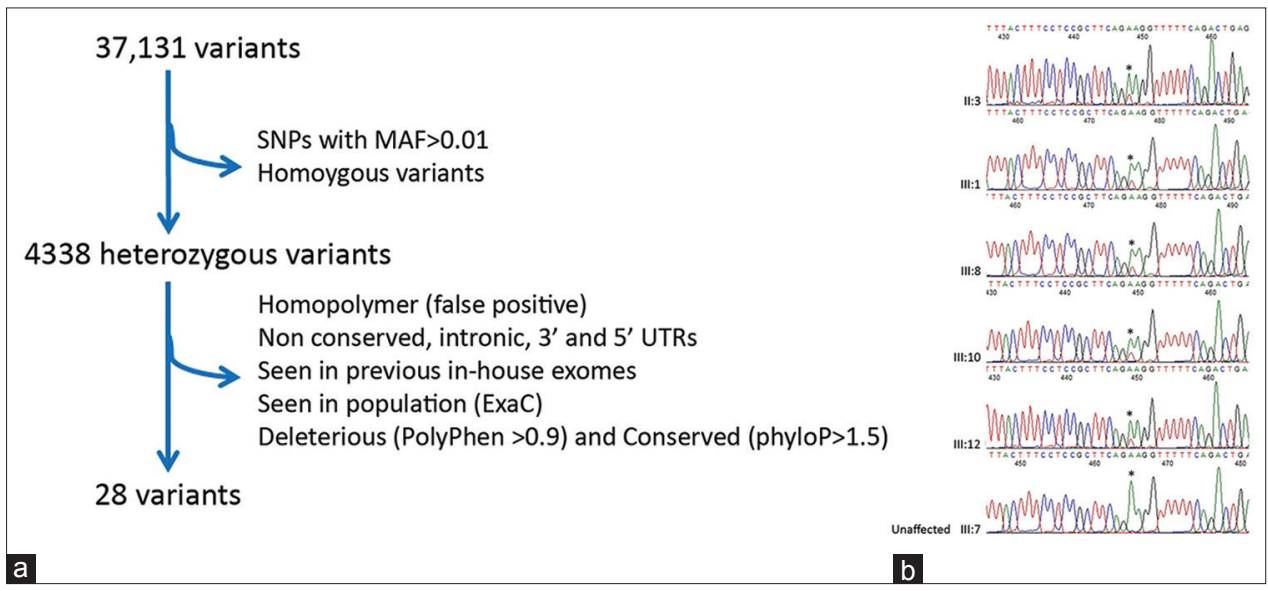

Figure 2: A nonsense mutation p.Lys900Ter in ATP2C1 causing HHD. (a) Whole exome sequencing results workflow. (b) Sanger sequencing results confirmed the segregation of the c.2698A>T mutation with HHD phenotype in 4 affected males and one female.

her late forties. To date, all individuals who have been reported as carriers of ATP2Cl mutations and present the complete clinical features of this disease. Even though HHD is transmitted as an autosomal 
Table 1: Primers used for Sanger sequencing.

\begin{tabular}{ll}
\hline Primer & Sequence \\
\hline Set1 Forward & 5' CACACAATTAGGTCCATTCTCCA 3' \\
Set1 Reverse & 5' TCATTCCTCACACCTACACAG 3' \\
Set2 Forward & 5' AGCGAATTCTCACTAATTGACCA 3' \\
Set2 Reverse & 5' TTCTGCTATTTGGTCAGACTGA 3' \\
\hline
\end{tabular}

dominant disease, variation in the pattern and the severity of symptoms within the same family have been reported [2]. The variability of age of onset and the different expressivity of the phenotype that we report here may provide evidence for genetic modifiers as well as for environmental factors such as sun, heat, stress and friction that affect the HHD development [2].

\section{CONCLUSION}

We report here a novel nonsense heterozygous mutation in ATP2Cl gene in 5 HHD-patients supporting that alterations in the human secretory pathway $\mathrm{Ca} 2+/ \mathrm{Mn} 2+$ ATPase are causative for this disease. It is worth noting that in this family one woman carries the nonsense ATP2C1 mutation but displays a mild phenotype of HHD. Our observations would thus be in line with variation in pattern and expressivity between male and female developing Hailey-Hailey disease phenotype or evidence for other modifiers. This aspect should be considered when providing genetic counselling to family members carrying such mutations.

\section{ACKNOWLEDGEMENTS}

We are grateful to the family's members for their contribution and participation in our study. We thank Sihem Sassi for her help on sample collection. M. Chourabi is a $\mathrm{PhD}$ student in the University of Monastir in Tunisia and is funded by an $A^{*}$ STAR Research Attachment Program. B. Reversade is a fellow of the Branco Weiss Foundation, an A*STAR Investigator and EMBO Young Investigator. This work was funded by the Skin Research Institute of Singapore and a Strategic Positioning Fund on Genetic Orphan Diseases from A*STAR, Singapore.

\section{REFERENCES}

1. Hailey H. Familial benign chronic pemphigus; report thirteen years after first observation of a new entity. South Med J. 1953;46:763-5.

2. Burge SM. Hailey-Hailey disease: the clinical features, response to treatment and prognosis. Br J Dermatol. 1992;126:275-82.

3. Kellermayer R. Hailey-Hailey disease as an orthodisease of PMR1 deficiency in Saccharomyces cerevisiae. FEBS Lett. 2005;579:2021-5.

4. Chave TA, Milligan A. Acute generalized Hailey-Hailey disease. Clin Exp Dermatol. 2002;27:290-2.

5. Metze D, Hamm H, Schorat A, Luger T. Involvement of the adherens junction-actin filament system in acantholytic dyskeratosis of Hailey-Hailey disease. A histological, ultrastructural, and histochemical study of lesional and non-lesional skin. J Cutan Pathol. 1996;23:211-22.

6. Hashimoto K, Fujiwara K, Harada M, Setoyama M, Eto H. Junctional proteins of keratinocytes in Grover's disease, Hailey-Hailey's disease and Darier's disease. J Dermatol. 1995;22:159-70.

7. Hu Z, Bonifas JM, Beech J, Bench G, Shigihara T, Ogawa H, et al. Mutations in ATP2C1, encoding a calcium pump, cause Hailey-Hailey disease. Nat Genet. 2000;24:61-5.

8. Sudbrak R, Brown J, Dobson-Stone C, Carter S, Ramser J, White J, et al. Hailey-Hailey disease is caused by mutations in ATP2C1 encoding a novel $\mathrm{Ca}(2+)$ pump. Hum Mol Genet. 2000;9:1131-40.

9. Callewaert G, Parys JB, De Smedt H, Raeymaekers L, Wuytack F, Vanoevelen J, et al. Similar $\mathrm{Ca}(2+)$-signaling properties in keratinocytes and in COS-1 cells overexpressing the secretory-pathway $\mathrm{Ca}(2+)-$ ATPase SPCA1. Cell Calcium. 2003;34:157-62.

10. Behne MJ, Tu CL, Aronchik I, Epstein E, Bench G, Bikle DD, et al. Human keratinocyte ATP2C1 localizes to the Golgi and controls Golgi Ca2+ stores. J Invest Dermatol. 2003;121:688-94.

11. Missiaen L, Raeymaekers L, Dode L, Vanoevelen J, Van Baelen K, Parys JB, et al. SPCA1 pumps and Hailey-Hailey disease. Biochem Biophys Res Commun. 2004;322:1204-13.

12. Kumar P, Henikoff S, Ng PC. Predicting the effects of coding nonsynonymous variants on protein function using the SIFT algorithm. Nat Protoc. 2009;4:1073-81.

13. Adzhubei IA, Schmidt S, Peshkin L, Ramensky VE, Gerasimova A, Bork P, et al. A method and server for predicting damaging missense mutations. Nat Methods. 2010;7:248-9.

14. Grantham R. Amino acid difference formula to help explain protein evolution. Science. 1974;185:862-4.

15. Pollard KS, Hubisz MJ, Rosenbloom KR, Siepel A. Detection of nonneutral substitution rates on mammalian phylogenies. Genome Res. 2010;20:110-21.

Copyright by Marwa Chourabi, et al. This is an open access article distributed under the terms of the Creative Commons Attribution License, which permits unrestricted use, distribution, and reproduction in any medium, provided the original author and source are credited.

Source of Support: M. Chourabi is a PhD student in the University of Monastir in Tunisia and is funded by an $A^{*}$ STAR Research Attachment Program. B. Reversade is a fellow of the Branco Weiss Foundation, an $A^{*}$ STAR Investigator and EMBO Young Investigator. This work was funded by the Skin Research Institute of Singapore and a Strategic Positioning Fund on Genetic Orphan Diseases from A*STAR, Singapore. Conflict of Interest: None declared. 